\title{
Possible Role of CDI Ia in Primary Immune Thrombocytopenia Patients on Immunosuppressive Therapy
}

This article was published in the following Dove Press journal: Journal of Blood Medicine

Muhamad R Abdel Hameed (D) ${ }^{1,2}$ Howiada A Nafady ${ }^{1,2}$

Mona Ibrahim Mostafa ${ }^{3}$

Douaa Sayed $^{4}$

Ahmad A Obiedallah (1D ${ }^{5}$

'Hematology Unit, Department of Internal Medicine, Assiut University Hospitals, Assiut University, Assiut, Egypt; ${ }^{2}$ Bone Marrow Transplantation Unit, South Egypt Cancer Institute, Assiut University, Assiut, Egypt; ${ }^{3}$ Department of Internal Medicine, Assiut General Hospital, Assiut, Egypt; ${ }^{4}$ Department of Clinical Pathology, South Egypt Cancer Institute, Assiut University, Assiut, Egypt; ${ }^{5}$ Critical Care Unit, Department of Internal Medicine, Assiut University Hospitals, Assiut University, Assiut, Egypt
Correspondence: Muhamad R Abdel Hameed

Hematology Unit, Department of Internal Medicine, Assiut University Hospitals, Assiut University, Assiut, 71515, Egypt

Tel +20 10975100I0

Email dr.muhamadramadan@yahoo.com
Background and Objectives: Immune thrombocytopenia (ITP) is one of the autoimmune diseases that presented by thrombocytopenia and increased risk of bleeding. Etiology of immune thrombocytopenia (ITP) is very complex. Lymphocyte function associated antigen-1 (LFA-1) plays important role in ITP. The aim of this study was evaluation of expression of CD11a on lymphocytes to explore its possible role in primary ITP patients also, regarding severity and response to immunosuppressive treatment.

Patients and Methods: This is a cross-sectional case-control study. Forty adult patients aged (18:58) years, 29 females and 11 males were enrolled as newly diagnosed primary ITP. Forty age and sex matched control subjects were randomly selected. The expression of CD11a on lymphocyte subpopulations (CD3+ T cells, CD3+CD4+ T cells and CD19+ B cells) was analyzed by flowcytometry at the start of the study and after 6 months of follow-up.

Results: The mean fluorescence intensity (MFI) of CD11a on CD3+ T and CD19+ B lymphocytes was significantly highly increased in ITP patients compared to healthy controls while MFI of CD11a on CD3+ CD4+Tclls was non-significant. MFI of CD11a on CD3+ and CD19+ B lymphocytes showed non-significant elevation with platelet count or bleeding score. MFI of CD11a on CD3+ showed significant highly increased level in refractory ITP compared with responder cases.

Conclusion: CD11a had possible role in the pathogenesis of ITP. Immunosuppressive therapy in ITP did not affect the level of CD11a expression on T and B lymphocytes. Levels of CD11a do not reflect the severity of ITP neither platelet count nor bleeding score. Increased MFI of CD11a in CD3+T lymphocytes of ITP patients may cause resistance to immunosuppressive therapy.

Keywords: Primary immune thrombocytopenia, immunosuppressive therapy, LFA-1, CD11a, B cells, T cells

\section{Background}

Immune thrombocytopenia (ITP) is an autoimmune disease characterized by thrombocytopenia with or without mucocutaneous bleeding. ${ }^{1}$ Antibody coated platelets in ITP, are destroyed by macrophages of the reticuloendothelial-system. ${ }^{2}$

Auto reactive B lymphocytes producing antiplatelet antibodies are the main etiology of ITP. Autoantibodies were not found in $30-40 \%$ of ITP patients. The antiplatelet autoantibodies are under the control of T cells. Although there are multiple drugs used in the treatment of ITP, still there are many refractory cases even after splenectomy, so 
there is a need to understand more about the pathogenesis of ITP to find other lines of treatment. ${ }^{3}$

Immune thrombocytopenia (ITP) is a significant clinical problem due to chronicity, treatment cost, occurrence mainly in, young, and relatively poorer quality of life than in people with other chronic diseases such as hypertension, arthritis, and cancer subjects. ${ }^{4}$ Most of the current therapeutic agents for ITP do not solve the fundamental problems that are responsible for the beginning and progression of the autoimmune process. Treatment strategy for ITP has focused on improving platelet life (either by immunosuppressive treatment or splenectomy) and increase platelet production. ${ }^{5}$

Abnormalities in the regulation of the immune system play an important role in the initiation and perpetuation of ITP. $^{6}$

Lymphocyte function associated antigen-1 (LFA-1) is belonging to the integrin family and consists of alpha chain CD11a and beta chain CD18 dimers, and expressed on the surface of T lymphocytes, B lymphocytes, macrophages, neutrophils, and monocytes. Its major ligand, intercellular adhesion molecule-1 (ICAM-1) is belonging to the immunoglobulin superfamily, on the surface of antigen-presenting cells (APCs). ${ }^{7}$

The combination of LFA-1 and ICAM-1 provide a coordinated signal and initiates lymphocyte activation, proliferation, and differentiation. Interaction of $\mathrm{T}$ cells with antigen-presenting cells (APCs), LFA-1, and ICAM1 participate in the formation of immunological synapse that promotes $\mathrm{T}$ cell proliferation and cytotoxicity. ${ }^{8}$

LFA-1 is similar to receptors such as CD4 and CD8. Disruption of LFA-1 activity strongly affects immune stability. ${ }^{9}$ CD11a is important for the generation of lymphoid progenitors in the BM that is essential to normal lymphocyte development. ${ }^{10}$

\section{Patients and Methods}

The current study is a cross-sectional case-control study conducted at Assiut University Hospital, Assiut, Egypt, from December 2017 to October 2019. Research Ethics Medical Review Board of Assiut University has approved the protocol (number 17200350). This study was conducted in accordance with the Declaration of Helsinki. An informed written consent was obtained from all subjects who participated in the study.

Forty newly diagnosed primary ITP patients aged 18:58 years (29 females and 11 males) attended the Clinical Hematology unit of Internal Medicine Department, Assiut
University Hospital, Assiut, Egypt were enrolled in the study. Diagnosis was done according to American Society of Hematology (ASH) guidelines. ${ }^{11,12}$

Exclusion criteria including:

1- Secondary cause of ITP as systemic lupus erythematous (SLE), viral infections (HIV, hepatitis B or C infections)

2-Underlying medical diseases that may cause thrombocytopenia as malignancy, megaloblastic anemia, aplastic anemia, lymphoproliferative disorders, liver disease, renal impairment, or pregnancy.

3-Organomegally and/or lymphadenopathy.

All patients in the study had full history taking, clinical examination, and assessment of bleeding according to ITP bleeding score (IBLS). All investigation for exclusions of secondary thrombocytopenia or other autoimmune diseases were done including rheumatoid factor, ANA and anti-double stranded DNA antibodies, Hepatitis B \&C, and HIV markers, liver function test, renal function, CMV IgM \& IgG, and Monospot test for infectious mononucleosis.

Expression of CD11a on lymphocyte subpopulations $(\mathrm{CD} 3+\mathrm{T}$ cells, $\mathrm{CD} 3+\mathrm{CD} 4+\mathrm{T}$ cells, and $\mathrm{CD} 19+\mathrm{B}$ cells $)$ were analyzed by flow cytometry at time of diagnosis.

Forty (age and sex matched) control subjects were randomly selected; they had no evidence of bleeding or autoimmune diseases. Females included in control group are non-pregnant. Informed consents were taken from all subjects.

All studied patients received initial treatment, dexamethasone $40 \mathrm{mg} /$ day for 4 days. ${ }^{12}$

During follow-up 8 patients showed insufficient elevation of platelet count and moderate bleeding continued after 4 weeks, we added Azathioprine $(150 \mathrm{mg} /$ day).

Five patients using rituximab $\left(375 \mathrm{mg} / \mathrm{m}^{2}\right.$ administered once weekly) with stopping steroid due to intolerable side effects after 9 weeks of using steroid including stress hyperglycemia and uncontrollable elevated blood pressure. One patient received cyclosporine (3-5 $\mathrm{mg} / \mathrm{kg} /$ day) after stopping steroid due to patient preference.

After 6 months all patients re-evaluated to detect response to therapy. The responders to treatment will comprise subjects with complete response (CR) or response (R). A platelet count of $\geq 100 \times 10^{9} / \mathrm{L}$ was interpreted as a complete response (CR), while response (R) was interpreted when platelets ranging from $30 \times 10^{9} / \mathrm{L}$ to less than $100 \times 10^{9} / \mathrm{L}$ with the duplication of the pretreatment platelet count. ${ }^{13}$ 
Second estimation for The expression of CD11a on lymphocyte subpopulations $(\mathrm{CD} 3+\mathrm{T}$ cells, $\mathrm{CD} 3+\mathrm{CD} 4+$ $\mathrm{T}$ cells, and CD19+ B cells) were analyzed by flow cytometry in patients how showed response to immunosuppressive therapy.

\section{Flow Cytometry Analysis}

The whole peripheral blood samples (anticoagulated with EDTA) were stained with the following antibodies; Fluorescein isothiocyanate FITC (Exbio) conjugated antiCD11a, CD3 V450, CD19 APC and CD4 PerCP Cy5.5 (B. D bioscience). A minimum of 10,000 events were acquired and analyzed by BD FACS Canto system (BD, San jose, CA). Appropriate isotype-matched controls were included in the experiments to identify positive populations. Data were analyzed with FASCDIVA software. The B (CD19+) and $\mathrm{T}(\mathrm{CD} 3+)$ lymphocytes were gated, expression of CD11a was detected on B and T-lymphocytes, total T-lymphocytes CD3+CD4+ (T-helper) cells were gated and expression of CD11a on them was detected before treatment (Figure 1) and after 6 months follow-up in those respond to treatment (Figure 2).

\section{Statistical Analysis}

Data were analyzed by version 24 of SPSS software (Chicago, IL, USA). The Shapiro-Wilk test for continuous variables was used as a measure of normality. Mean \pm standard deviation (SD) was used to express parametric variables, independent $t$-test was used to compare means of level of CD11a between patients and control and also used to compare means between patients who respond and who did not respond to immunosuppressive therapy while paired $t$-test was used to compare means of level of CD11a in patients who respond (before and after treatment) and Pearson's correlation coefficients were calculated to explore associations between level of CD11a in patients and severity of disease at presentation according to platelet count and IBLS (ITP bleeding score). P value $<0.05$ was considered significant.

\section{Results}

Ages of included patients ranged from 18 to 58 years with a mean $\pm \mathrm{SD}=31.70 \pm 10.96$ years. Female patients were $29(72.5 \%)$ and 11 patients (27.5\%) were males.

The platelet count of patients at presentation was 2 $\times 10^{9} / \mathrm{L}: 30 \times 10^{9} / \mathrm{L}$. All patients showed skin bleeding (purpura and ecchymosis), 2 patients (5\%) were presented by skin bleeding only while 4 patients $(10 \%)$ were presented by skin and nasal bleeding (epistaxis) and 2 patients $(5 \%)$ were presented by skin, nasal and oral bleeding. Vaginal bleeding was the main presentation, in 20 patients $(50 \%$ of all patients $(68.96 \%$ of female patients) and was associated with other types of bleeding. Vaginal bleeding was associated with skin bleeding in 11 patients $(27.5 \%)$ and was associated with skin and oral bleeding in 4 patients $(10 \%)$ while associated with skin and nasal bleeding in 5 patients $(12.5 \%)$. Urinary bleeding associated with skin bleeding in $1(2.5 \%)$ patient and associated with skin and oral bleeding in 2 patients $(5 \%)$, while rectal bleeding was reported in 2 patients $(5 \%)$ (Figure 3 ). The severity of bleeding was variable and presented by ITP bleeding score ranged between 2 and 11 .

All patients in the study received immunosuppressive therapy which included steroid alone (26 patients $65 \%$ ), steroid + azathioprine ( 8 patients $20 \%$ ), rituximab after stopping steroid (5 patients $12.5 \%$ ) and cyclosporine (1 patient $2.5 \%$ ).

Follow-up after 6 months we reported that thirty patients respond to immunosuppressive therapy (75\%) while 10 patients $(25 \%)$ were refractory for treatment. The platelets count follow-up after 6 months in patients responding to treatment was $190.50 \pm 59.19 \times 110^{9} / \mathrm{L}$. While platelets count before treatment was $15.46 \pm 8.17 \times 110^{9} / \mathrm{L}$ with highly significant $(p$ value $=0.000$ ). Platelets count before treatment in Patients non-responder to treatment was $19.30 \pm 7.81 \times 110^{9} / \mathrm{L}$. While, it was 28.88 $\pm 8.94 \times 110^{9} / \mathrm{L}$ after treatment with significant $(p$ value $=$ 0.02) (Table 1).

Forty healthy control were included in the study, their ages were ranged between 18 and 51 years old with a mean $\pm \mathrm{SD}=33.92 \pm 10.30$, Females control were 30 $(75 \%)$ and none of them were pregnant at the time of the study. Males control were 10 (25\%) All control had normal platelet count ranged from 150 to $387 \times 110^{9} / \mathrm{L}$.

The mean fluorescence intensity (MFI) of CD11a was detected by flowcytometry in T-lymphocytes (CD3+ cells), T helper (CD3+CD4+ cells), and B-lymphocytes (CD19+) in patients and control (Table 2). There was a statistically highly significant difference between cases and control in MFI of CD11a in CD3+ cells (MFI in patients 70.15 +23.88 while in control $46.85+17.50$ ) with more expression in patients than control. Also, there was a statistically highly significant difference between cases and control in MFI of CD11a in CD19+ cells (MFI in patients 65.20 \pm 32.36 while in control $44.54 \pm 21.02$ with $p$ value $=$ 0.001) with more expression in patients than control. 

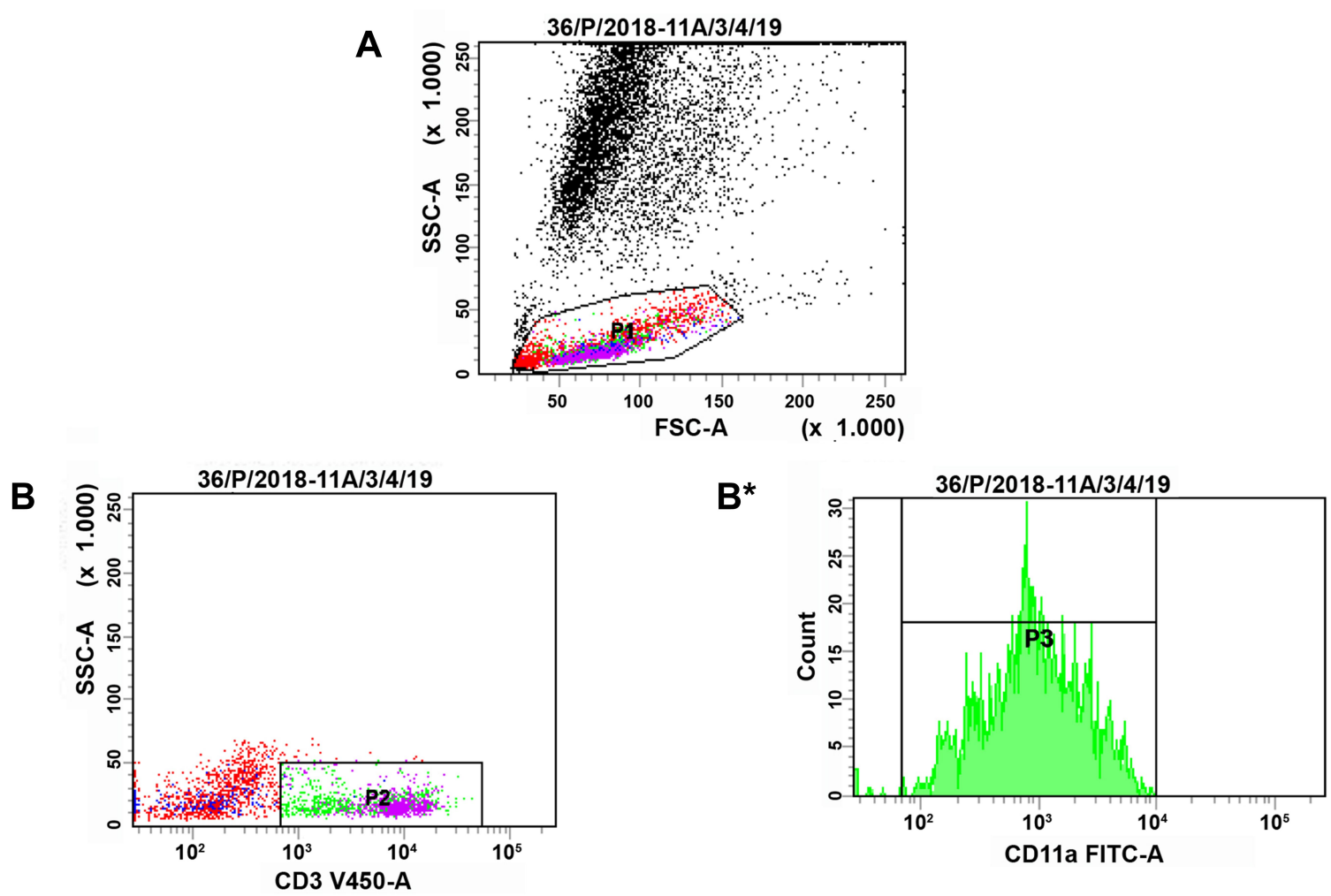

C

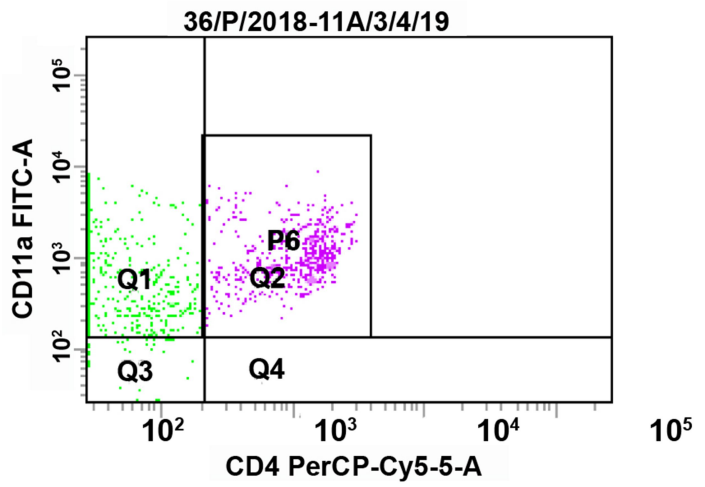

D

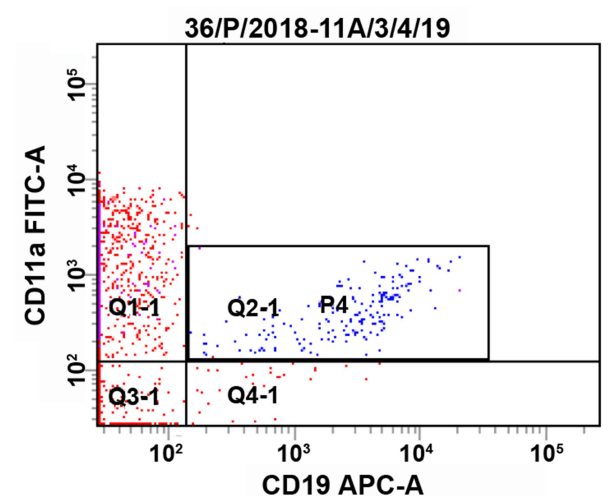

C*

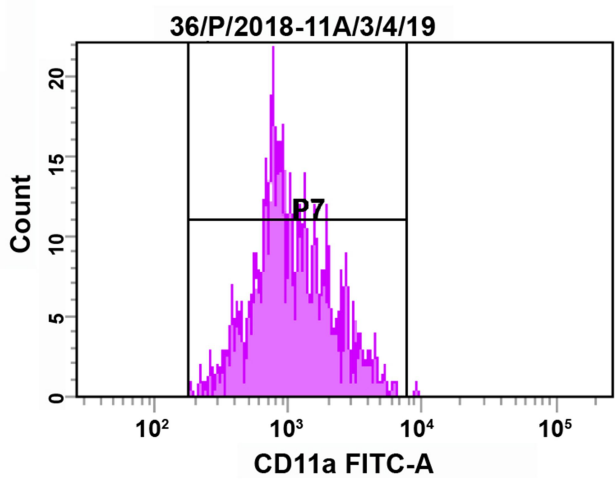

D*

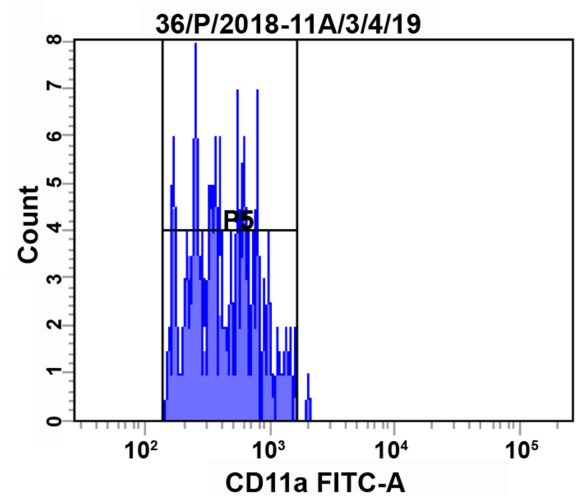

Figure I The expression of CDI la on lymphocyte subsets in ITP patient before treatment. (A) Lymphocytes were gated by flow cytometry (PI). (B) CD3+ cells gated by flow cytometry (P2). (B*) MFI of CDI la gated on CD3+ cells (P3). (C) Expression of CDI la on CD3+CD4+ T cells (P6). (C*) MFI of CDI la gated on CD3+CD4+ T cells (P7). (D) Expression of CDI la on CDI9+ cells (P4). (D*) MFI of CDI la gated on CDI9+ cells (P5). 

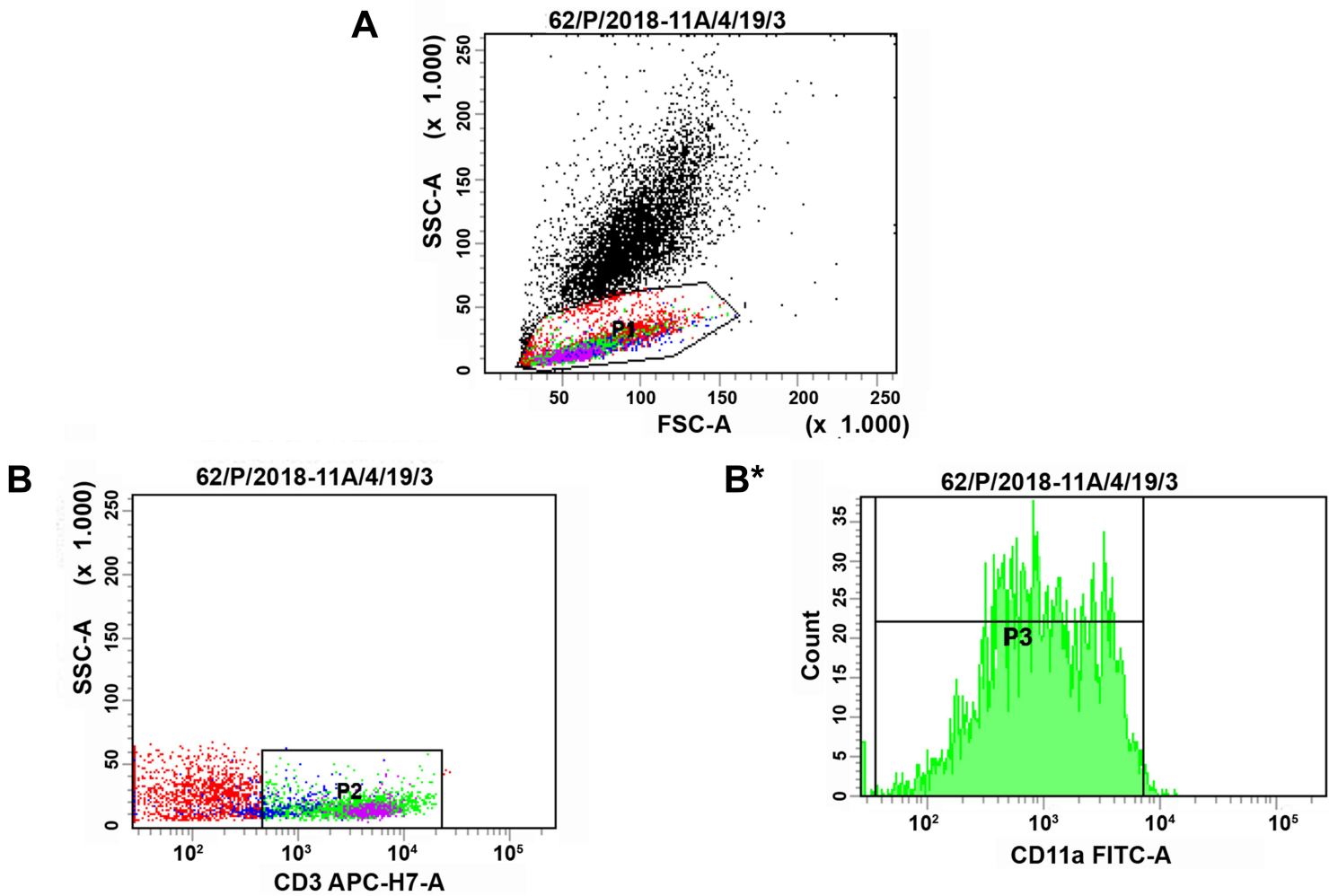

C

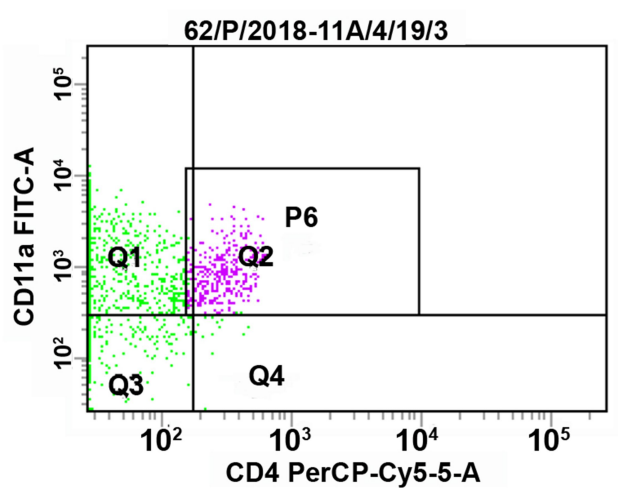

$\mathbf{C}^{*}$

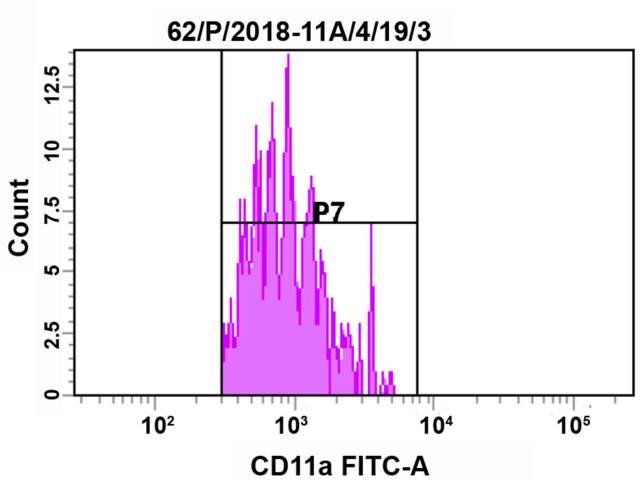

D

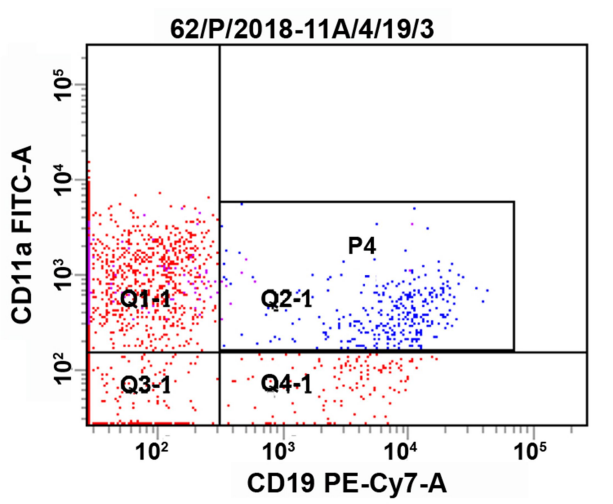

D*

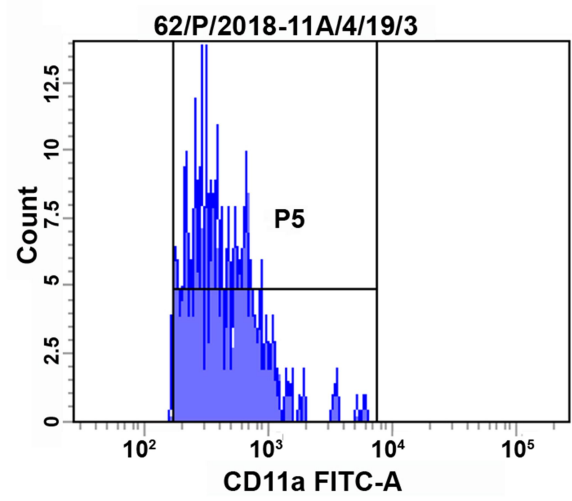

Figure 2 The expression of CDI la on lymphocyte subsets after 6 months follow-up in ITP patient whose response to treatment. (A) Lymphocytes were gated by flow cytometry (PI). (B) CD3+ cells gated by flow cytometry (P2). (B*) MFI of CDI la gated on CD3+ cells (P3). (C) Expression of CDI la on CD3+CD4+ T cells (P6). (C*) MFI of CDI la gated on CD3+CD4+ T cells (P7). (D) Expression of CDI la on CDI9+ cells (P4). (D*) MFI of CDI la gated on CDI9+ cells (P5). 


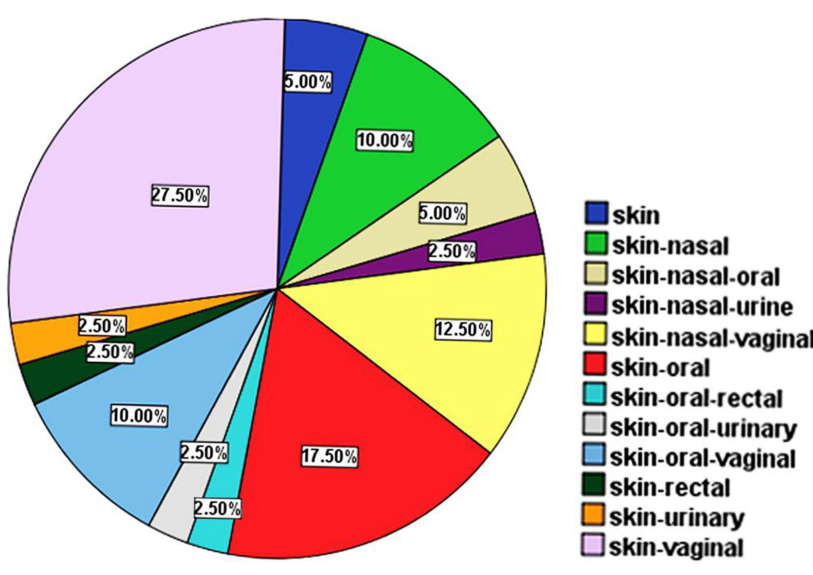

Figure 3 Pattern of bleeding in ITP patients at presentation.

There was no significant difference in the MFI of CD11a in $\mathrm{CD} 3+\mathrm{CD} 4+$ cells between patients and control (78.56 \pm 34.04 and $70.13+22.45$, respectively) with $p$-value $=0.19$.

At presentation, no significant correlation between MFI of CD11a in CD3 + cells with platelet count $(\mathrm{p}$ value $=$ $0.61, r=0.07)$ or with bleeding score $(\mathrm{p}=0.79, \mathrm{r}=0.02)$. Also there was no significant correlation between MFI of
CD11a in CD19+ cells with platelet count $(\mathrm{p}$ value $=0.54$, $\mathrm{r}=17)$ or with bleeding score $(\mathrm{p}=0.71, \mathrm{r}=0.04)$ (Table 3$)$.

All patients in the study received immunosuppressive therapy and after follow-up for 6 months we found that 30 patients respond to immunosuppressive therapy $(75 \%)$ while 10 patients $(25 \%)$ were refractory for treatment.

There was no statistically significant difference in the MFI of CD11a in CD19+ cells between patients who responded and not responded to immunosuppressive therapy ( $p=0.50$ ) while there was a highly significant difference in MFI of CD11a in CD3+ cells between patients who responded and not responded to therapy $(p=0.000)$ with higher MFI in patients who did not respond to immunosuppressive therapy (Table 4).

There was no significant difference in the MFI of CD11a in CD19+ and CD3+ cells between patients before and after response to treatment with $p$ - value $=0.16$ and 0.19 , respectively (Table 5).

\section{Discussion}

In this study 40 patients diagnosed as primary ITP were included after exclusion of secondary causes for

Table I Platelet Count Changes Before and After Treatment

\begin{tabular}{|l|c|c|}
\hline Group & $\begin{array}{c}\text { Platelet Count Before Treatment } \\
\text { Mean + SD }\end{array}$ & $\begin{array}{c}\text { Platelet Count After } 6 \text { Month of Treatment } \\
\text { Mean + SD }\end{array}$ \\
\hline Responders & $15.46+8.17$ & $190.50+59.19$ \\
\hline Non-responders & $19.30+7.81$ & $0.000 * * *$ \\
\hline P value & $0.2 *$ & $0.02 * *$ \\
\hline
\end{tabular}

Notes: *Non-significant. **Significant. ***Highly significant.

Table 2 Difference Between Mean of CDI la Level in Patients and Control

\begin{tabular}{|l|l|l|l|}
\hline & Case Mean + SD & Control Mean + SD & Significant (2-Tailed) \\
\hline CD I la MFI in CD3+ cells & $70.15 \pm 23.88$ & $46.85 \pm 17.50$ & $0.000^{* * * *}$ \\
\hline CD Ila MFI in CDI9+ cells & $65.20 \pm 32.36$ & $44.54 \pm 21.02$ & $0.00 I^{* * * *}$ \\
\hline CD I la MFI in CD3+CD4+ cells & $78.56 \pm 34.04$ & $70.13 \pm 22.45$ & $0.19 *$ \\
\hline
\end{tabular}

Notes: *Non-significant $(\mathrm{P}$ value $>0.05)$. $* * *$ Highly significant $(\mathrm{P}$ value $<0.005)$.

Table 3 Correlation of CDI la with Platelet Count and Bleeding Score

\begin{tabular}{|l|l|l|}
\hline Correlation & PLT Count & Bleeding Score \\
\hline$C D ~ I I a$ in CDI9+ cells & $P=0.54 * r=0.17$ & $P=0.7 I^{*} r=0.04$ \\
\hline$C D$ Ila in CD3+ cells & $P=0.6 I^{*} r=0.07$ & $P=0.79 * r=0.02$ \\
\hline
\end{tabular}

Note: ${ }^{*}$ Non-significant $(P$ value $>0.05)$. 
Table 4 Response of Patients to Immunosuppressive Therapy

\begin{tabular}{|l|l|l|l|}
\hline & N & CD I Ia in CD3+ Cells & CD I Ia in CD I9+ Cells \\
\hline Refractory & $10(25 \%)$ & $79.47 \pm 18.25$ & $71.33 \pm 32.53$ \\
\hline Response & $30(75 \%)$ & $42.18 \pm 15.43$ & $63.16 \pm 32.60$ \\
& P value & $0.000^{* *}$ & $0.50 *$ \\
\hline
\end{tabular}

Notes: *Non-significant $(\mathrm{P}$ value $>0.05)$. **Highly significant $(\mathrm{P}$ value $<0.005)$.

Table 5 Difference Between Level of CDI la Before and After Treatment in Patients Who Respond to Treatment

\begin{tabular}{|l|l|l|}
\hline Group & CD I Ia in CD3+ Cells Mean + SD & CD I Ia in CDI9+ Cells Mean + SD \\
\hline Before treatment & $79.47 \pm 18.25$ & $63.16 \pm 32.60$ \\
\hline After treatment & $69.44 \pm 24.55$ & $43.59 \pm 21.26$ \\
\hline P value & $0.19^{*}$ & $0.16^{*}$ \\
\hline
\end{tabular}

Note: *Non-significant $(\mathrm{P}$ value $>0.05)$.

thrombocytopenia. Most of the patients were females. Other studies showed that ITP is more common in females. $^{14,15}$

The bleeding pattern of patients in this study was bleeding from different body orifices and skin but without dangerous bleeding, this confirming that ITP is rarely associated with life-threatening bleeding events as stated by Michel and his colleagues. ${ }^{16}$

The results of the present study provide insight into the possible role of (LFA-1) that represented by CD11a in the pathogenesis of ITP by comparing its MFI on T and B lymphocytes and T-helper cells between newly diagnosed ITP patients and healthy control and results showed that there was high statistically significant difference between cases and control in the MFI of CD11a in T-cells (CD3+ cells) ( $p$ value $=0.000)$ with more expression in patients than control confirming LFA-1 plays an important role on $\mathrm{T}$ cell activation and increased CD11a expression on $\mathrm{T}$ cells may induce auto reactivity in ITP. Also there was a highly significant difference in the level of CD11a in CD19+ cells $(p$ value $=0.001)$ with more expression in patients than control so we suspected that increase expression of CD11a on CD19+ cells in ITP could result in sufficient B cell stimulatory signals that lead to activation of B cells to produce autoantibodies. Results of this study were confirming the theory of Carrasco and his colleagues, that LFA-1/ICAMs interaction leads to biochemical signals for T-cell-dependent $\mathrm{B}$ cell activation and antibody production and decreases the threshold of $\mathrm{B}$ cell activation caused by facilitating $B$ cell adhesion and synapse formation. $^{17}$
Results of this study were agreed with the study of Sela who detect an important role of LFA-1/ICAMs interaction in the pathogenesis of SLE. Using LFA-1 monoclonal antibodies in lupus mice reduce the production of autoantibodies; reduce the development of autoimmune reaction, and the symptoms of lupus nephritis improved. ${ }^{18}$

In the current study, there was no significant difference between MFI of CD11a on T-helper lymphocytes (CD3 + CD4+ cells) and this may indicate that T-helper may have no role in the pathogenesis of ITP. There was no significant correlation between level of CD11a in CD3+ and CD19+ and platelet count and bleeding score so the CD11a expressions on $\mathrm{B}$ and $\mathrm{T}$ lymphocyte were not correlated with the platelet counts or bleeding score in ITP, indicating that the expression levels of CD11a on lymphocyte cells can not reflect the severity of the disease. These previous results agreed with the result Chinese study by Liu. ${ }^{19}$

Patients with primary ITP in this study received immunosuppressive therapy including, steroid, azathioprine, rituximab, and cyclosporine according to the international consensus report and evidence-based practice guideline by the American Society of Hematology, the standard therapy for primary ITP includes corticosteroids as the first-line agent. $^{20}$

Follow-up after 6months, response achieved in 30 patients while the other 10 patients were refractory to immunosuppressive therapy and need other lines of treatment. Comparing means of level of CD11a in CD3+ and CD19+ cells between patients according to response to immunosuppressive therapy showed no significant 
difference between responders and refractory patients in the MFI of CD11a in CD19+ cells while there was a highly significant difference in expression of CD11a in CD3+ cells between patients with more expression in patients who could not achieve response to immunosuppressive therapy, this reflect that excessive expression of CD11a in T-lymphocytes can increase the possibility of resistance to immunosuppressive therapy so, the level of CD11a in T-lymphocytes in ITP patients can be used to expect a response to treatment. Within responders to treatment, comparing means of level of CD11a in CD3+ and CD19+ cells before and after response to immunosuppressive therapy showed no significant difference which indicates that immunosuppressive therapy did not affect LFA1/ICAM-1 pathway and using LFA-1 monoclonal antibodies may provide different mechanism for controlling ITP. A study by Sela showed that using LFA-1 monoclonal antibodies in lupus mice leading to decrease the production of autoantibodies and stopping the development of autoimmune reaction, and the symptoms of lupus nephritis resolved. ${ }^{18}$ A study by Stephen and his colleagues detected the possible role of LFA-1/ICAM-1 interaction in the pathophysiology of dry eye disease (DED) as one of the autoimmune diseases. LFA-1/ICAM-1 interaction is a rational therapeutic target in DED. Inhibition of LFA-1/ ICAM-1 interaction represents a rational targeted approach in treating DED. ${ }^{21}$

\section{Conclusions}

LFA-1 and ICAM-1 play a role in the pathogenesis of ITP. Immunosuppressive therapy in ITP did not affect the level of CD11a expression on $\mathrm{T}$ and B lymphocytes. Levels of CD11a do not reflect the severity of ITP neither platelet count nor bleeding score. Increased MFI of CD11a in CD3 $+\mathrm{T}$-lymphocytes of ITP patients may cause resistance to immunosuppressive therapy.

\section{Author Contributions}

All authors made a significant contribution to the work reported, whether that is in the conception, study design, execution, acquisition of data, analysis and interpretation, or in all these areas; took part in drafting, revising or critically reviewing the article; gave final approval of the version to be published; have agreed on the journal to which the article has been submitted; and agree to be accountable for all aspects of the work.

\section{Funding}

Due to limited resources, this work was self-funded by the authors.

\section{Disclosure}

All authors declare that they have no conflicts of interest for this work.

\section{References}

1. McMillan R. Immune-mediated thrombocytopenias focus on chronic immune thrombocytopenic purpura. Semin Hematol. 2007;44:1-2. doi:10.1053/j.seminhematol.2007.11.001

2. Ahn YS, Horstman LL. Idiopathic thrombocytopenic purpura: pathophysiology and management. Int J Hematol. 2002;76(2):123-131. doi:10.1007/BF03165102

3. Kawana M, Ikeda Y. The role of autoreactive T Cells in the pathogenesis of idiopathic thrombocytopenic purpura. Int J Hematol. 2005;81:106-112. doi:10.1532/IJH97.04176

4. Kim SY, Solomon DH. Tumor necrosis factor blockade and the risk of viral infections. Nat Rev Rheumatol. 2010;6(3):165-174. doi:10.1038/nrrheum.2009.279

5. Targownik LE, Bernstein CN. Infectious and malignant complications of TNF inhibitor therapy in IBD. Am J Gastroenterol. 2013;108 (12):1835-1842. doi:10.1038/ajg.2013.294

6. McKenzie CG, Guo L, Freedman J, Semple JW. Cellular immune dysfunction in immune thrombocytopenia (ITP). $\mathrm{Br} J$ Hematol. 2013;163:10-23. doi:10.1111/bjh. 12480

7. Ghislin S, Obino D, Middendorp S, Boggetto N, Alcaide-Loridan C, Deshayes FLFA. 1 and ICAM-1 expression induced during melanomaendothelial cell co-culture favors the transendothelial migration of melanoma cell lines in vitro. BMC Cancer. 2012;12:455. doi:10.1186/1471-2407-12-455

8. Parameswaran N, Suresh R, Bal V, Rath S, George A. Lack ofICAM1 on APCs during $\mathrm{T}$ cell priming leads to poor generation of central memory cells. J Immunol. 2005;175:2201-2211. doi:10.4049/ jimmunol.175.4.2201

9. Pribila JT, Quale AC, Mueller KL, Shimizu Y. Integrins and T cell mediated immunity. Annu Rev Immunol. 2004;22:157-180. doi:10.1146/annurev.immunol.22.012703.104649

10. Bose TO, Colpitts SL, Pham QM, Puddington L, Lefrançois L. CD11a is essential for normal development of hematopoietic intermediates. $J$ Immunol. 2014;193:2863-2872. doi:10.4049/jimmunol.1301820

11. Neunert C, Lim W, Crowther M. The American Society of Hematology 2011 evidence-based practice, guidelines for immune thrombocytopenia. Blood. 2011;117:4190-4207. doi:10.1182/blood2010-08-302984

12. Neunert C, Terrell DR, Arnold DM, et al. American Society of Hematology 2019 guidelines for immune thrombocytopenia. Blood Adv. 2019;10(23):3829-3866. doi:10.1182/bloodadvances.2019000966

13. Rodeghiero F, Stasi R, Gernsheimer T, et al. Standardization of terminology, definitions and outcome criteria in immune thrombocytopenic purpura of adults and children: report from an international working group. Blood. 2009;113:2386-2393. doi:10.1182/blood2008-07-162503.

14. Abbasi SY, Milhem M, Zaru L. A positive antinuclear antibody test predicts for a poor response to initial steroid therapy in adults with idiopathic thrombocytopenic purpura. Ann Hematol. 2008;87(6):459462. doi:10.1007/s00277-008-0448-1

15. Grimaldi-Bensouda L, Nordon C, Michel M, et al. Immune thrombocytopenia in adults: a prospective cohort study of clinical features and predictors of outcome. Haematologica. 2016;101(9):1039-1045. doi:10.3324/haematol.2016.146373. 
16. Rosenblum MD, Remedios KA, Abbas AK. Mechanisms of human autoimmunity. J Clin Invest. 2015;125(6):2228-2233.

17. Carrasco YR, Fleire SJ, Cameron T, Dustin ML, Batista FD. LFA-1/ ICAM-1 interaction lowers the threshold of B cell activation by facilitating $\mathrm{B}$ cell adhesion and synapse formation. Immunity. 2004;20:589-599. doi:10.1016/S1074-7613(04)00105-0

18. Sela U, Mauermann N, Hershkoviz R, et al. The inhibition of autoreactive $\mathrm{T}$ cell functions by a peptide based on the CDR1 of an antiDNA autoantibody is via TGF-beta mediated suppression of LFA-1 and CD44 expression and function. J Immunol. 2005;175:7255-7263. doi:10.4049/jimmunol.175.11.7255
19. Liu YX, Zhang F, Yao QM, Yuan T, Xu J, Zhu XJ. Expression of CD11a in lymphocyte subpopulation in immune thrombocytopenia. Int J Clin Exp Pathol. 2015;8(12):15642-15651.

20. Provan D, D M A, J B B, et al. Updated international consensus report on the investigation and management of primary immune thrombocytopenia. J Blood Adv. 2019;3:3780-3817.

21. Stephen C, Pflugfelder MS, Steven Z, Amir SLFA. 1/ICAM-1 interaction as a therapeutic target in dry eye disease. J Ocular Pharmacol Ther. 2017;33(1). doi:10.1089/jop.2016.0105

\section{Publish your work in this journal}

The Journal of Blood Medicine is an international, peer-reviewed, open access, online journal publishing laboratory, experimental and clinical aspects of all aspect pertaining to blood based medicine including but not limited to: Transfusion Medicine; Blood collection, Donor issues, Transmittable diseases, and Blood banking logistics; Immunohematology; Artificial and alternative blood based therapeutics; Hematology; Biotechnology/nanotechnology of blood related medicine; Legal aspects of blood medicine; Historical perspectives. The manuscript management system is completely online and includes a very quick and fair peer-review system. Visit http://www.dovepress.com/testimonials.php to read real quotes from published authors. 